\title{
The Current Distribution of Symmetrical Dual and Triple Feeding Full-Wave Dipole Antenna
}

\author{
Yahya S. H. Khraisat \\ Electrical and Electronics Department $\backslash$ Al-Huson University College \\ Al-Balqa Applied University, Jordan, PO box 1375, Irbed 21110 \\ E-mail: yahya@huson.edu.jo \\ Khedher A Hmood \\ School of Electrical \& Electronic Engineering \\ University Sains Malaysia, Pulau Pinang \\ E-mail: khedher100@yahoo.com \\ Al-Mofleh Anwar (Corresponding author) \\ Department of Electrical Engineering / Faculity of engineering Technology \\ Al-Balqa' Applied University Amman, Jordan Amman, PO box 15008, marka ashamalia \\ E-mail: anwaralmofleh@yahoo.com
}

Received: Semptember 7, 2011

Accepted: October 21, $2011 \quad$ Published: December 1, 2011

doi:10.5539/mas.v5n6p126

URL: http://dx.doi.org/10.5539/mas.v5n6p126

\begin{abstract}
This paper is focused on the analysis of the current distribution of symmetrical full-wave dipole antenna based on double and triple feeding techniques; the current distributions were measured using a shielded loop protruding through a slit in the antenna surface along its axis, gives exact and accurate measurement rather than sinusoidal approximation. However, the sinusoidal distributions of current and voltage are approximations rather than exact descriptions. Next the current distributions were modeled and formulated whose parameters were deduced from the measured current distribution by applying the current distribution data in models as derived by IEEE standard.
\end{abstract}

Keywords: Full wave, Dipole antenna, Dual and triple feeding and symmetrical

\section{Introduction}

Practically, the input techniques impedance, the far-field radiation pattern and the directive gain are indispensable parameters in characterizing the antenna. However, the calculation and measurement of the current distributions are important for the analysis and design of antennas because the other properties of the antenna may be determined from the current distribution. The far-field radiation pattern of a microwave antenna is usually evaluated from the measured near field. Similarly, in the case of a linear antenna, the radiation pattern may be estimated from the current distribution which is measured more easily than the far field (Yahya, etal., 2008; Harrington, 1960; Egashira, et al., 1985).

\section{Measurement Method}

This section presents results from an experimental investigation of current distribution test. This investigation serves to experimentally find the current distribution wave form, and to obtain formulas by using curve fitting and try and error. The relative magnitude of the current distribution along the antennas was measured and normalized with respect to maximum amplitude. Measurements were made at $5 \mathrm{~cm}$ interval along the antenna's arm using loop type current probe (Collin, 1955; IEEE, Standard, 1980; Yaghjian, 1986; King, 1956). As shown in Figure 1, two-shielded loop were used namely fixed shielded loop and moving shielded loop. The fixed shielded loop was used as reference and is placed in the centre of the dipole while moving shielded loop was used to measure the current distribution along the antenna axis. The two shielded loop were connected to vector 
analyzer. This method may be used when the radius of dipole is rather thick, thus is impossible for a very thin antenna. The diameter should be sufficiently large for the surface currents to be considered (Collin, 1955; IEEE, Standard, 1980; Yaghjian, 1986; King, 1956). When a shielded loop is located near a test antenna; the electromotive force (EMF) on the loop is induced by the whole current on the antenna.

To evaluate the performance of a shielded loop, a system error ratio need to be defined as the ratio of the output current due to a unit electric field parallel to the loop to the output current due to a unit magnetic field through the loop. For a circular loop of diameter $0.01 \lambda$, the error ratio measured by them was about $-30 \mathrm{~dB}$ (King, 1956; Harrington, 1976; Hassan, 1987).

In this paper, a circular loop of diameter $0.08 \lambda$ made from copper was used in all current distribution measurements. The measurements were conducted firstly on half wave dipole, centre tap full wave, and off centre full wave dipole in which their characteristics are well known. Initially, the current distribution was measured, followed by the measurement of parameters of antenna like gain, radiation patterns and input impedance. The relative magnitude of the current distribution along the antennas was measured and normalized with respect to maximum amplitude.

\section{Results}

\subsection{Symmetrical Dual Feeding}

For simplicity, the constructed antenna was named with respective reference as shown in Table 1. For Antenna A1, the measured current distribution is indicated in Figure2. From Figure2, Curve fitting, trial and error were used to obtain the Equation.1, corresponding to a conventional formulation of single feeding. Figure 2 shows a plot of $I(z)$ with z representing the predicted current distribution of Antenna A1.

$$
I(z)=\sin \beta\left(\frac{L}{2}-/ z /\right)
$$

Where:

$I(z)$ - is the current at any point on the antenna at $\mathrm{z}$ direction,

$\beta$ - is the phase constant,

$\beta=\frac{2 \pi}{\lambda}$

$\lambda$ - is wavelength in meter,

L- is the length of the antenna element and the expressions are assumed to be valid for $-\frac{L}{2} \leq z \leq \frac{L}{2}$, so that the antenna element straddles the $\mathrm{x}$ y-plane

For Antenna A2, the measured current distribution is indicated in Figure 3 which led to Equation 2 and representing the current distribution for Antenna A2. Figure3 shows the predicted current distribution $I(z)$, as a function of $\mathrm{z}$.

\subsection{Triple Feeding}

$$
I(z)=\sin \left(\beta\left(\frac{L}{2}-z\right)\right.
$$

For Antenna C1, C2, and C3, the measured current distribution is indicated in Figures 4, 5 and 6 respectively. Curve fitting and trial and error were used to obtain Equations 3, 4, and 5. Figure 4 shows the predicted current distribution of Antenna C1, Figures 5 shows the predicted current distribution for Antenna C2 while those of Figure 6 correspond to Antenna C3.

$$
\begin{gathered}
I(z)=\sin \beta\left(\frac{L}{2}-|z|\right)+\cos \left(\beta \cdot \frac{z}{2}\right) \\
I(z)=\sin \beta\left(\frac{L}{2}-|z|\right)+0.5 \cos \left(\beta \cdot \frac{z}{2}\right) \\
I(z)=\sin \beta\left(\frac{L}{2}-|z|\right)+0.1 \cos \left(\beta \cdot \frac{z}{2}\right)
\end{gathered}
$$

For triple feeding, Equation 3, Equation 4, and Equation 5 are similar except that the cosine coefficient factor (M) 
is different; thus, the three Equations can be generalized into applicable for the triple feeding as depicted in Equation 6.

$$
I(z)=\sin \beta\left(\frac{L}{2}-|z|\right)+M \cos \left(\beta \cdot \frac{z}{2}\right)
$$

The current distribution of Antenna A1 was compared with the simulated current distribution of Hallen and King, and the radius of the Antenna A1 was set for different values. However the current distribution of Antenna A1 is correspond to the Orfanidis's simulation. The current distribution is assumed to be symmetric with respect to the antenna centre where the feed point is located. It was reported that the Pocklington current is not required to vanish at the last sampling point, like the Hallen case (Yaghjian, 1986; King, 1956).

This condition was not incorporated into the Pocklington Equation. Nevertheless, the Pocklington currents tend to zero at the antenna end-points as $\mathrm{M}$ becomes larger, where $\mathrm{M}$ is number of samples (Harrington, 1976; Hassan, 1987; King, 1981). Figures 7, 8 and 9 show the simulated current distribution of Hallen numerical solution, King's 3-term, and predicted current of Antenna A1. It also shows the effect of the radius of the wire on the characteristics of the current distribution. When the radius is very small, King's approximation incorporates the conditions $I\left( \pm \frac{L}{2}\right)=0$

For antenna A2, the Equation of the current distribution of antenna A1 reduces to $I(z)=\sin \left(\beta\left(\frac{L}{2}-z\right)\right)$; this represents the characteristics of current distribution of Antenna A2.

Antenna A2 has similar characteristics of full-wavelength antenna as composed of two half-wavelength antennas having identical radiating properties, one excited positively and the other negatively, or $180^{\circ}$ out of phase, which exhibits maxima along the positive and negative z-axis. It was reported that the current distribution approximation is valid only when the load impedance is small or is equal to the complex conjugate of the antenna feed-point impedance (Khamas, et al., 1997; Werner, 1998; Best, 2002). However the measured input impedance of the antenna is small.

\section{Conclusion}

New methods to enhance the gain of the full wave dipole antenna were introduced. It was demonstrated that a full wave antenna, when fed with a multi feed, shows a high performance at resonance frequency. This can be exploited to achieve a high gain. Expressions for the current distribution of full-wave dipole antennas were developed. The relation of the current distribution with the feeding modifications and the polarity of feeding were numerically and experimentally investigated. The expressions for current distribution were developed, and validated, and compared with the experimental results.

For symmetrical dual feeding, the Equation of the current distribution of Antenna A1 is found to be similar to the conventional full-wave antenna Equation. For A2, the Equation follows the theoretical concept of the full-wave antenna (off-centre fed). However, the Symmetrical feeding in-phase achieved $3.82 \mathrm{dBi}$ gains.

\section{References}

Best, S. R. (2002). A comparison of the performance properties of the Hilbert curve fractal and meander line monopole antennas. Microwave Opt. Technol. Letter, 35(4), 258-262. http://dx.doi.org/10.1002/mop.10576

Collin, R. E. (1955). Theory and Design of Wide- Band Multisection Quarter-Wave Transformers, Proc. IRE. 43, 179.

Egashira, S., Taguchi, M., \& Kitajima, H. (1985), The effect of the end surface current on the numerical solution of wire antennas. Trans. IECE Japan, J68-B (6), 714-721.

Harrington, R. F. (1960). Effect of antenna size on gain, bandwidth, and efficiency, J. res. NBS. 64D, 1.

Harrington, R. F. (1967). Matrix Methods for Field Problems. Proc. IEEE. 55, 136. http://dx.doi.org/10.1109/PROC.1967.5433

Hassan, S. I. S. (1987). Matching in Rhombic and Pseudo Rhombic Antennae. PhD Thesis, University of Exeter. IEEE. (1980). Stanadrd Test Procedure for Antenna. ANSI/IEEE. 149-1979. IEEE Press. New York.

Khamas, S. K., Cook, G. G., Waldron, R. J., \& Edwards, R. M. (1997). Moment method analysis of printed single-arm wire spiral antennas using curved segments. IEE Proceedings-Microwaves Antennas and Propagation, 144, 261-265. http://dx.doi.org/10.1049/ip-map:19971195 
King, R .W. P., \& Smith .(1981). Antenna in Matter. Cambridge .MA: MIT Press.

King, R.W. P. (1956). Theory of the Center Driven Square Loop Antenna. IRE Transactions on Antennas and Propagation, AP-4(4), 393. http://dx.doi.org/10.1109/TAP.1956.1144431

Werner, D. H. (1998). A method of moments approach for the efficient and accurate modeling of moderatelythick cylindrical wire antenna. IEEE Trans. Antennas Propagat, 46, 373-382. http://dx.doi.org/10.1109/8.662656

Yaghjian, A. D. (1986). An Overview of Near-Field Antenna Measurements. I EEE Electromagnetic wave and Propagation, AP-34, (1), 30-45.

Yahya, S. H., Khraisat, K. A. H., \& Anwar, A. (2008). Analysis of the Parameters of Asymmetrical Dual Feeding Full Wave Dipole Antenna. Microwave and Remote Sensing Symposium. September (22-24), 215-217.

Table 1. A specifications and references of full wave dipole antennas

\begin{tabular}{|c|c|c|}
\hline Antenna No & Characteristics & References \\
\hline 1 & Symmetrical Dual Feeding in phase & Antenna A1 \\
\hline 2 & Symmetrical Dual Feeding out of phase & Antenna A2 \\
\hline 3 & Symmetrical Triple Feeding (a, $b, \mathrm{c}$ in phase) & Antenna C1 \\
\hline 4 & $\begin{array}{c}\text { Symmetrical Triple Feeding (a, } \mathrm{b} \text { in phase),(c out of } \\
\text { phase })\end{array}$ & Antenna C2 \\
\hline 5 & $\begin{array}{c}\text { Symmetrical Triple Feeding (a, } \mathrm{c} \text { in phase),(b out of } \\
\text { phase) }\end{array}$ & Antenna C3 \\
\hline
\end{tabular}

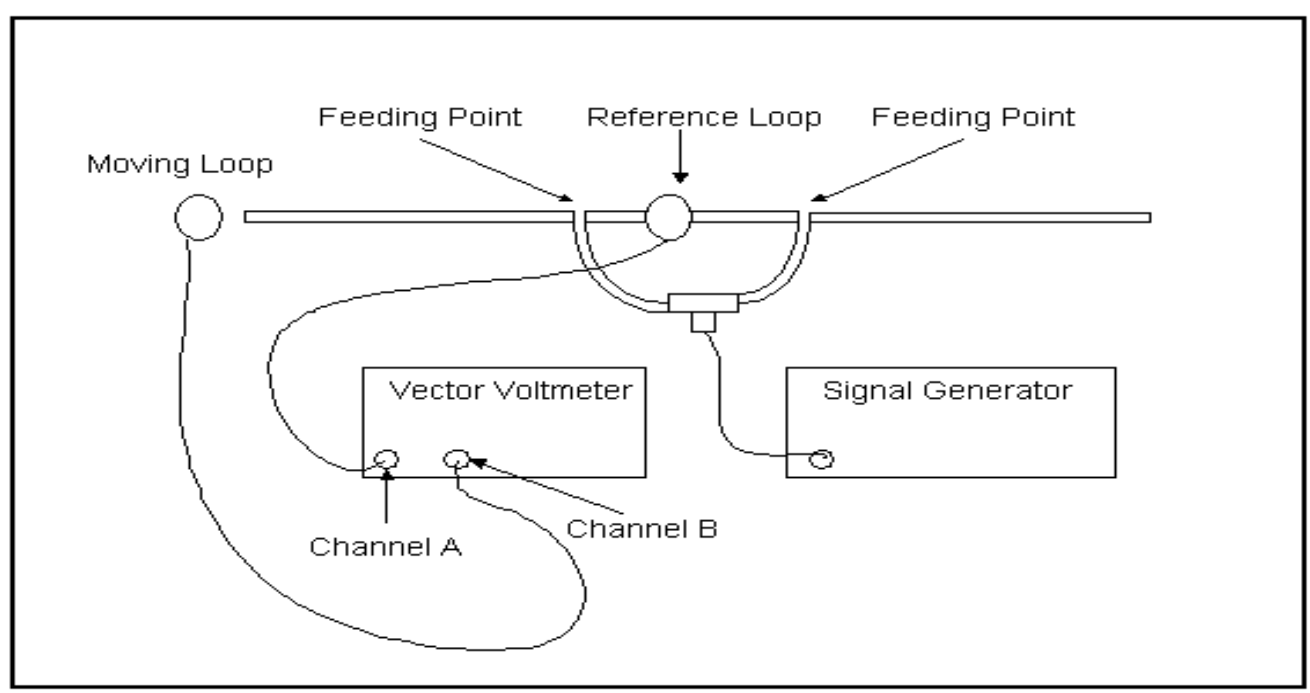

Figure 1. Measurements of current distribution using two shielded loops 


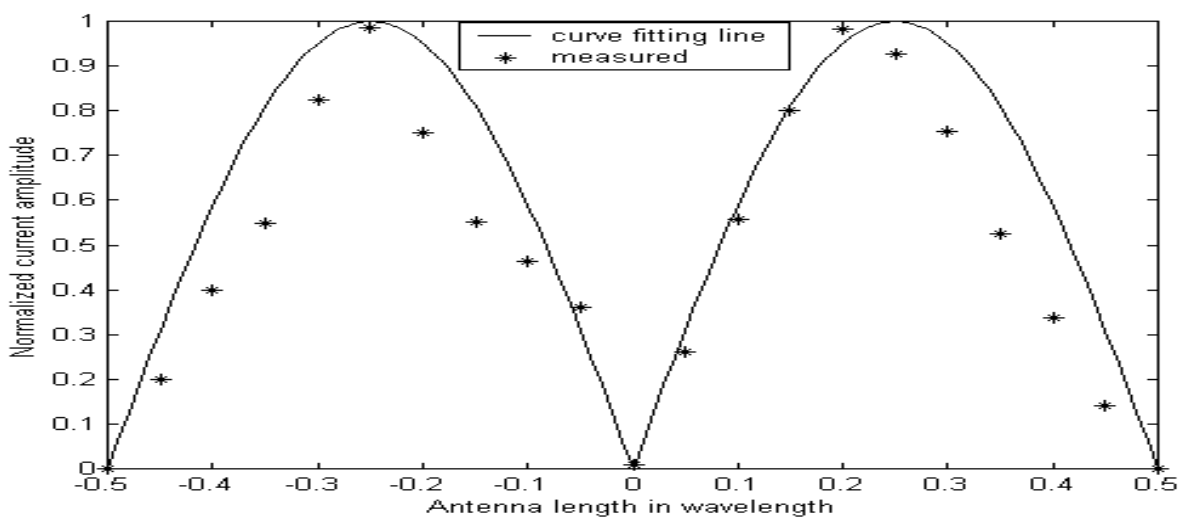

Figure 2. Measured and predicted current distribution for Antenna A1

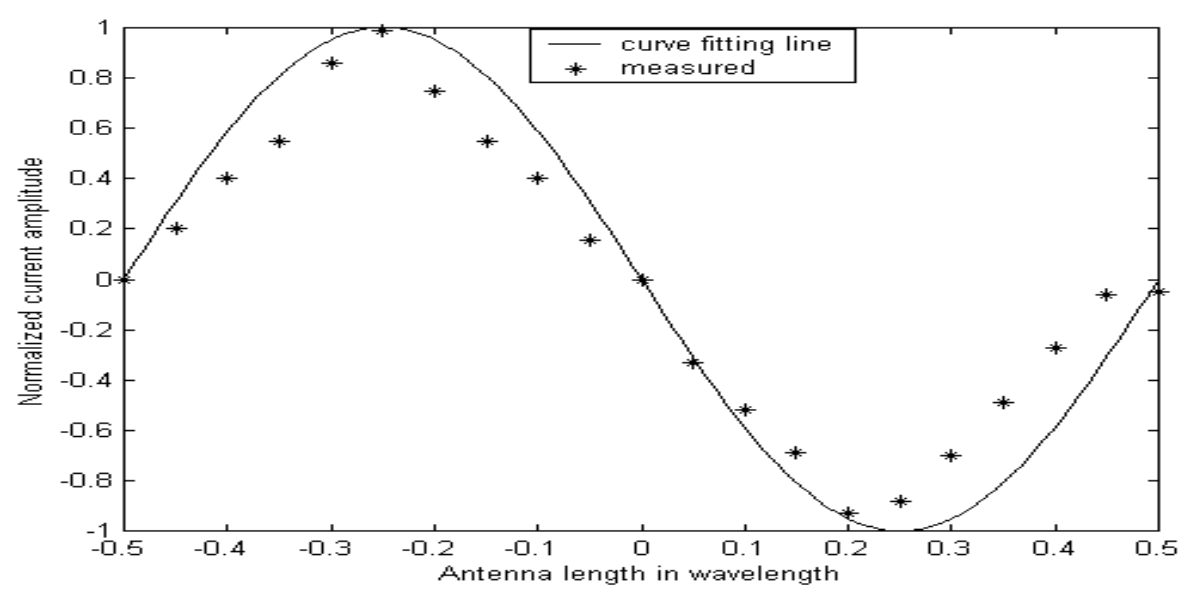

Figure 3. Measured and predicted current distribution for Antenna A2

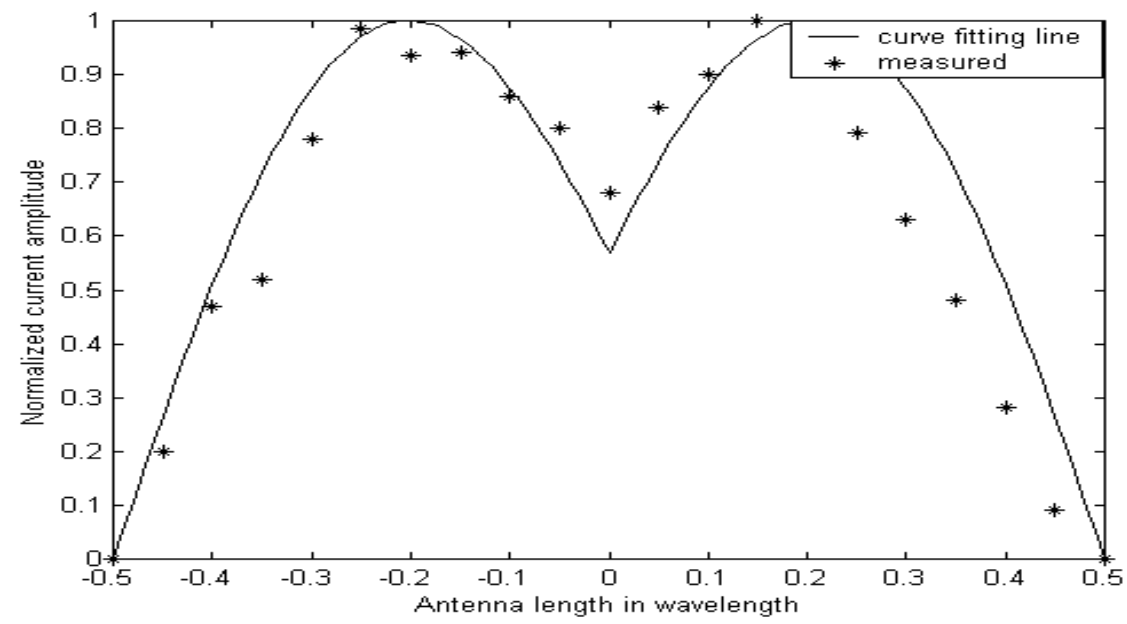

Figure 4. Measured and predicted current distribution for Antenna C1 


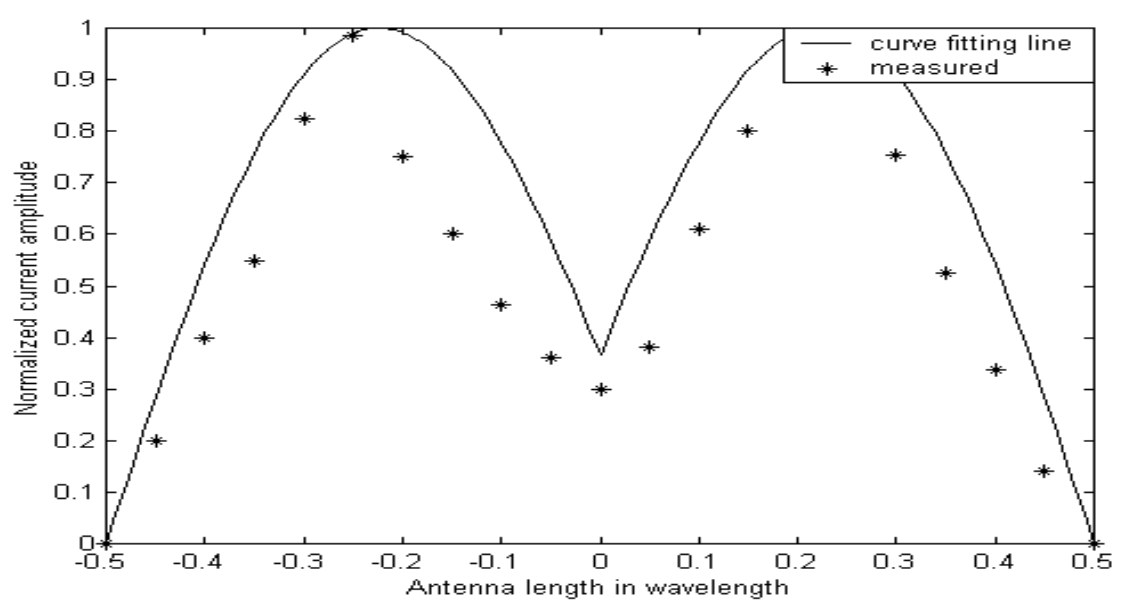

Figure 5. Measured and predicted current distribution for Antenna C2

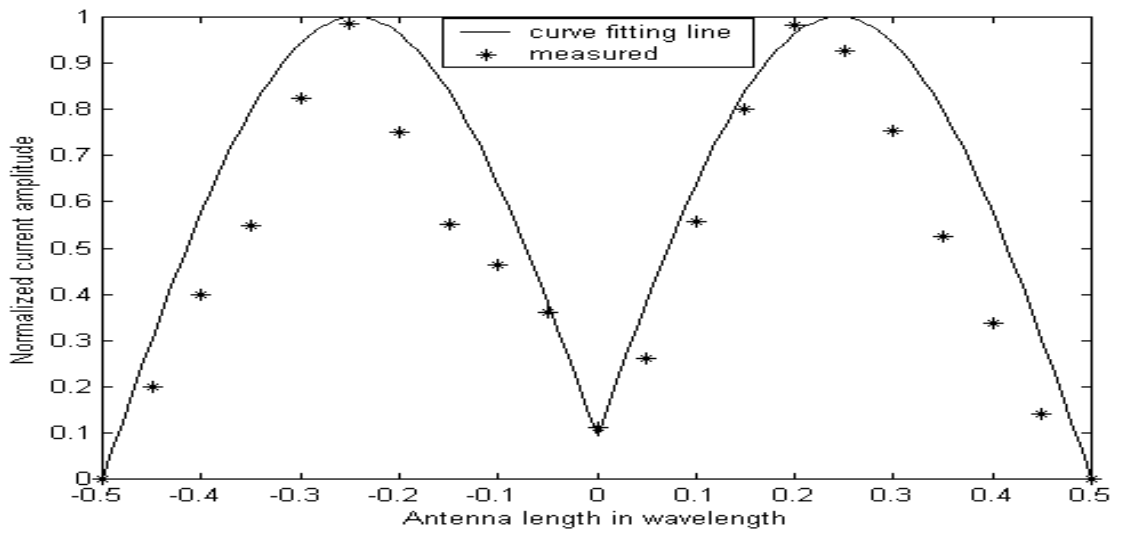

Figure 6. Measured and predicted current distribution for Antenna C3

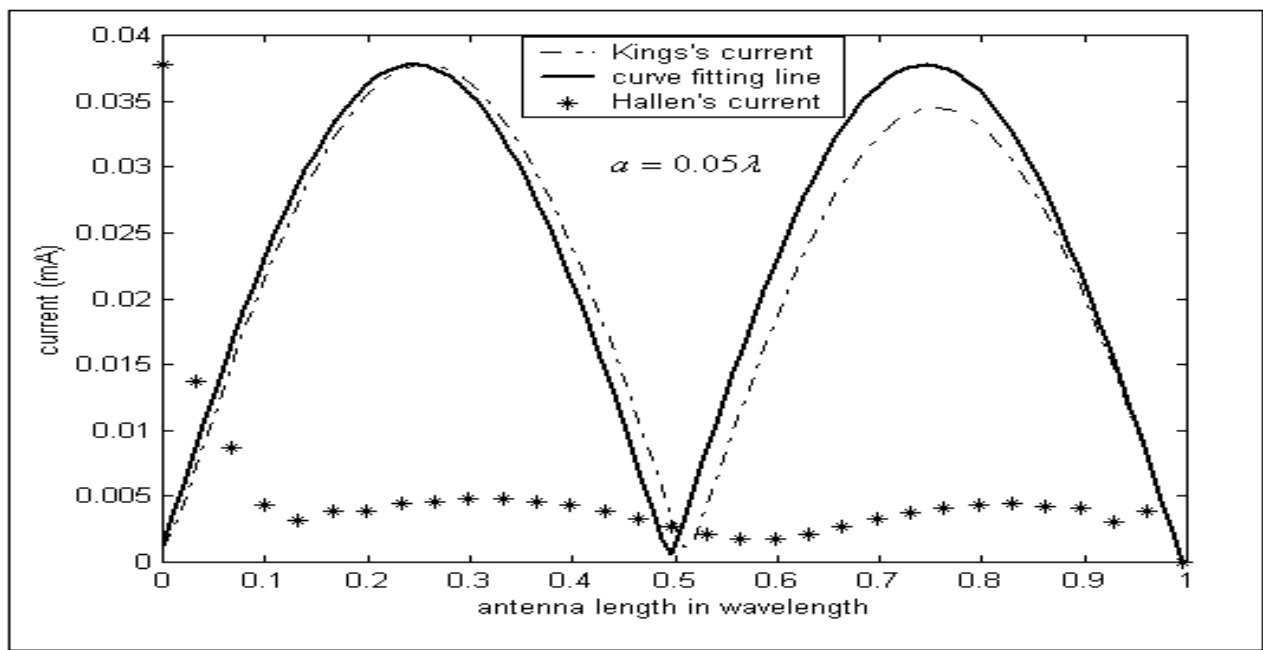

Figure 7. Comparison between the simulated currents, and predicted current of Antenna A1 


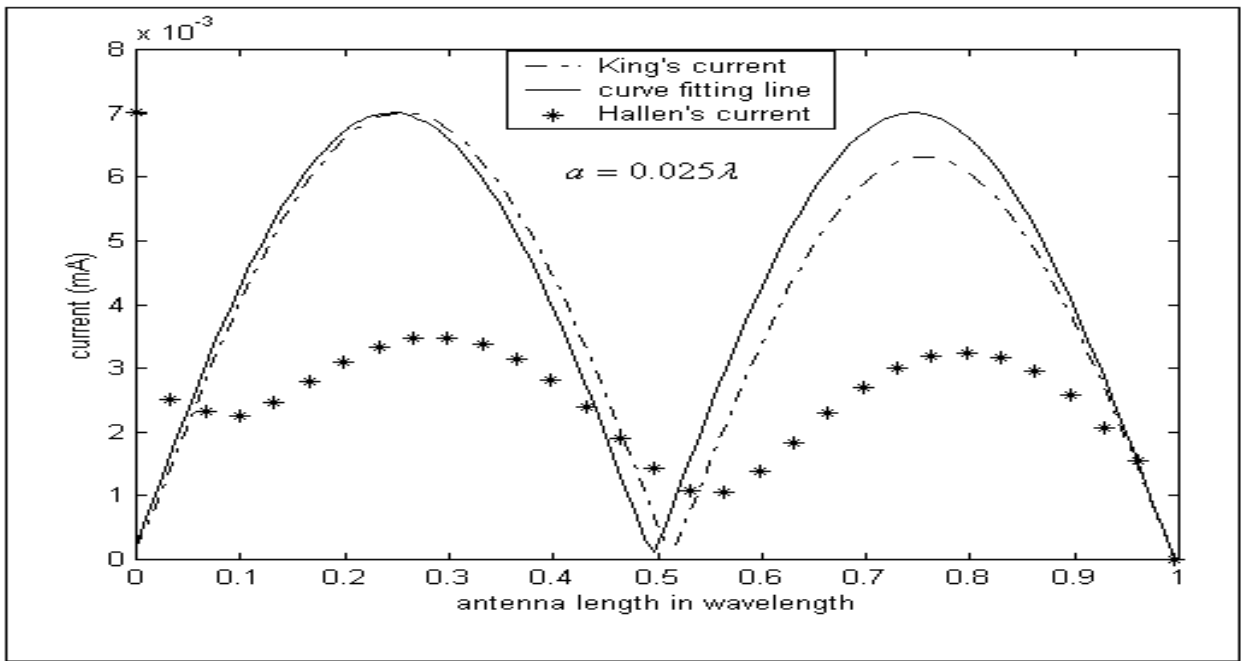

Figure 8.Comparison between the simulated currents and predicted Current of Antenna A1

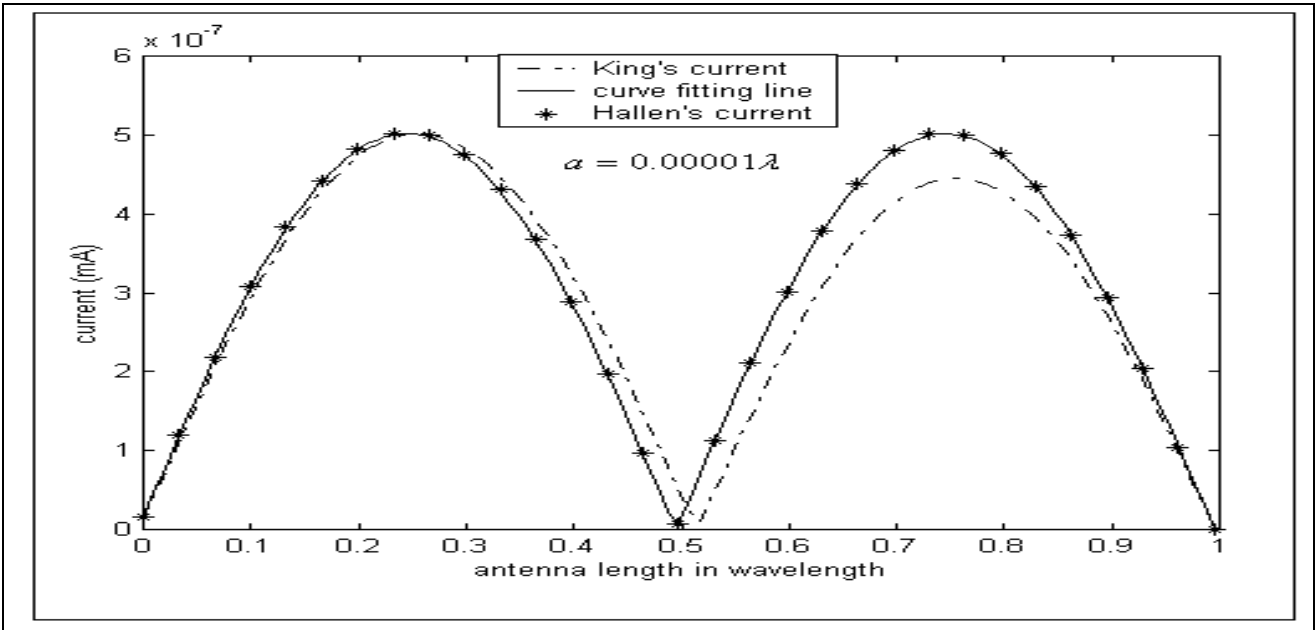

Figure 9. Comparison between the simulated currents, and predicted

\section{Current of Antenna A1}

